\title{
Total flavonoid fraction of the Herba epimedii extract suppresses urinary calcium excretion and improves bone properties in ovariectomised mice
}

\author{
Wen-Fang Chen ${ }^{1,2} \dagger$, Sao-Keng Mok $^{1,3} \dagger$, Xin-Luan Wang ${ }^{4}$, Ka-Hang Lai ${ }^{1,3}$, Wan-Ping Lai ${ }^{1,3}$, \\ Hon-Kit Luk ${ }^{1,3}$, Ping-Chung Leung ${ }^{5}$, Xin-Sheng Yao ${ }^{4,6 *}$ and Man-Sau Wong ${ }^{1,3 *}$ \\ ${ }^{1}$ Department of Applied Biology and Chemical Technology, The Hong Kong Polytechnic University, Kowloon, Hong Kong \\ ${ }^{2}$ State Key Disciplines: Physiology (in incubation), Medical College of Qingdao University, Qingdao, People's Republic of \\ China \\ ${ }^{3}$ State Key Laboratory of Chinese Medicine and Molecular Pharmacology, Shenzhen, People's Republic of China \\ ${ }^{4}$ College of Traditional Chinese Materia Medica, Shenyang Pharmaceutical University, Shenyang 110016, People's Republic \\ of China \\ ${ }^{5}$ Department of Orthopaedics and Traumatology, The Chinese University of Hong Kong, Shatin, Hong Kong \\ ${ }^{6}$ Institute of Traditional Chinese Medicine and Natural Products, College of Pharmacy, Jinan University, Guangzhou \\ 510632, People's Republic of China
}

(Received 20 January 2010 - Revised 16 July 2010 - Accepted 26 July 2010 - First published online 6 September 2010)

\begin{abstract}
Flavonoids are the active components of Herba epimedii (HEP), a commonly used herb for the management of osteoporosis in China over the centuries. The present study aims to characterise the in vivo effects of its total flavonoid (TF) fraction on bone properties and mineral metabolism as well as to study the mechanism involved in achieving its protective effects against ovariectomy (OVX)-induced bone loss. TF suppressed OVX-induced increase in urinary Ca excretion as well as loss of bone mass and strength at the distal femur in mice in a dosedependent manner. The changes in urinary Ca excretion were inversely correlated with the expressions of renal Ca transport protein (CaBP-28K) and vitamin D receptor mRNA in OVX mice. TF $(100 \mu \mathrm{g} / \mathrm{g})$ treatment prevented the deterioration of trabecular bone microarchitecture induced by OVX in mice. In addition, TF treatment increased the expression of type I collagen and osteocalcin mRNA and the ratio of osteoprotegerin/receptor activator of NF- $\mathrm{BB}$ ligand mRNA, and suppressed the increase in IL- 6 mRNA induced by OVX in the femur of mice. The present results indicate that the optimal dosage of the TF fraction of HEP for the improvement of bone properties and mineral metabolism in OVX mice was between 50 and $100 \mu \mathrm{g} / \mathrm{g}$. Mechanistic studies indicated that TF might increase renal Ca reabsorption, stimulate the process of osteoblast formation as well as suppress the process of osteoclastogenesis in OVX mice.
\end{abstract}

Key words: Herba epimedii: Total flavonoids: Osteoporosis: Ovariectomised mice

The Women's Health Initiative as well as the Million Women Study indicated that oestrogen or hormone replacement therapy increased the risk of postmenopausal women to develop breast cancer, stroke, thrombosis and $\mathrm{CVD}^{(1-3)}$. These findings have led to the advice that hormone replacement therapy should not be considered first-line therapy for the prevention of osteoporosis. As existing therapeutic agents, such as bisphosphonates, selective oestrogen receptor modulators, teriparatide and calcitonin $^{(4)}$, are either of high cost or associated with different side effects, alternative approaches for the prevention or treatment of osteoporosis are worth exploring.

Herba epimedii (HEP) is commonly used in traditional Chinese medicine for 'strengthening the kidney' ${ }^{, 5,6)}$ and nourishes the bone. HEP is one of the most frequently prescribed herbs in traditional Chinese medicine formula for the management of osteoporosis in China. Studies have shown that HEP extract could reduce bone loss in an ovariectomised (OVX) rat model ${ }^{(7-11)}$ as well as promote cell proliferation and increase alkaline phosphatase activity in

\footnotetext{
Abbreviations: BMD, bone mineral density; CaBP-28K, Ca transport protein; $\mathrm{E}_{2}, 17 \beta$-oestradiol; GAPDH, glyceraldehyde-3-phosphate dehydrogenase; HEP, Herba epimedii; OPG, osteoprotegerin; OVX, ovariectomy; RANKL, receptor activator of NF-кB ligand; SSI, stress-strain index; TF, total flavonoid; VDR, vitamin D receptor.
}

*Corresponding author: Dr M.-S. Wong, fax +852 23649932, email bcmswong@polyu.edu.hk; X.-S. Yao, email tyaoxs@jnu.cn tThese authors contributed equally to the studies. 
primary rat calvarial osteoblasts ${ }^{(12-18)}$. A recent study also demonstrated that it can promote the osteogenic differentiation of human bone marrow-derived mesenchymal stem cells ${ }^{(19)}$. Our previous study indicated that HEP total extract could increase trabecular bone mineral density (BMD) in ovariectomised rats and stimulate osteoblastic cell proliferation and differentiation in UMR 106 cells. HEP total extract could also induce the expression of osteoprotegerin (OPG, a soluble, decoy receptor that binds receptor activator of NF- $\mathrm{KB}$ ligand (RANKL) mRNA expression) and the ratio of OPG to RANKL (a membrane-bound TNF ligand family) expression in UMR 106 cells, suggesting that it could modulate the process of osteoclastogenesis ${ }^{(20)}$.

A recent study by Zhang et $a l^{(21)}$ demonstrated that a preparation containing $60 \mathrm{mg}$ icariin (the major flavonoid compound in HEP), $15 \mathrm{mg}$ daidzein and $3 \mathrm{mg}$ genistein could reduce bone loss in late postmenopausal women in a 24-month randomised, double-blind and placebocontrolled trial. This study provides evidence to support that the flavonoid compounds in HEP might account for the bone-protective effects of HEP in vivo. Furthermore, numerous in vitro ${ }^{(22-24)}$ and in vivo ${ }^{(25)}$ studies showed that icariin could stimulate osteoblastic cell functions, suppress osteoclastic activities and improve bone mineral density and bone strength in ovariectomised rats. Our recent study further suggests that the anabolic effects of icariin on bone might be mediated through ligand-independent activation of oestrogen receptor ${ }^{(24)}$. These studies further indicate that flavonoid compounds are the active components in HEP extract, which account for its bone-protective effects in vivo. However, the optimal dosage and the mechanism of actions by which flavonoid compounds in HEP exert bone-protective effects in vivo are not clear.

In the present study, we aim to characterise the dosedependent effects of the total flavonoid (TF) fraction of HEP extract on bone and mineral metabolism in ovariectomised mice as well as to elucidate the mechanism of actions involved in mediating its bone-protective effects in vivo.

\section{Materials and methods}

Preparation of total flavonoids fraction from Herba epimedii total extract

HEP (E. Koreanum Nakai) was collected in June 2003-July 2003 in a valley in Xinbin, Liaoning Province, and authenticated by Q.S. Sun, Professor of Pharmacognosy, Shenyang Pharmaceutical University, China. A voucher specimen (no. 19980816) has been deposited in the Institute of Traditional Chinese Medicine and Natural Products of Jinan University, China. The total extract was subjected to water extraction and column chromatography to yield four fractions with different polarities, namely water, 30\%, 50\% and 95\% ethanol. The fractions of 50 and $95 \%$ ethanol were pooled to give the TF fraction. The preparation was filtered and concentrated under vacuum to produce a powder at a yield of $9.5 \%$. The TF fraction was stored at room temperature before use. Fig. 1 shows a typical chromatographic profile of the TF fraction of the HEP total extract. HPLC analysis has been performed with standard compounds using the same elution procedure as that used with the TF extract of HEP. Peaks in the profile of TF with the same retention time with authentic markers (epimedin B, icariin, caohuoside $\mathrm{E}$ and baohuoside I) were identified.

\section{Animals}

Experimental protocols and procedures were approved by the Institutional Animal Care and Use Committee of The Hong Kong Polytechnic University. Female C57BL/6J mice were purchased from the Laboratory Animal Services Centre (the Chinese University of Hong Kong, HK).

Expt 1: dose-dependent effects of total flavonoids fraction of Herba epimedii extract in ovariectomised mice

Experimental groupings. One-month-old C57BL/6J mice were randomly separated into seven groups including Sham + vehicle (Sham, $n$ 8), OVX + vehicle (OVX, $n$ 8), OVX $+17 \beta$-oestradiol $\left(\mathrm{E}_{2}, 4 \mu \mathrm{g} / \mathrm{g}\right.$ per $\left.\mathrm{d}, n 8\right), \mathrm{OVX}+\mathrm{TF}$ (TF50, $50 \mu \mathrm{g} / \mathrm{g}$ per d, $n$ 8), OVX + TF $(\mathrm{TF} 100,100 \mu \mathrm{g} / \mathrm{g}$ per $\mathrm{d}, n 8), \mathrm{OVX}+\mathrm{TF}(\mathrm{TF} 200,200 \mu \mathrm{g} / \mathrm{g}$ per $\mathrm{d}, n$ 8) and $\mathrm{OVX}+\mathrm{TF}(\mathrm{TF} 400,400 \mu \mathrm{g} / \mathrm{g}$ per $\mathrm{d}, n$ 8). The animals were either ovariectomised or sham operated. After recovery from surgery for $18 \mathrm{~d}$, they were orally administered with vehicle, $\mathrm{E}_{2}$ or TF for 6 weeks. The animals were fed with diet containing $0.6 \% \mathrm{Ca}$ and $0.65 \% \mathrm{P}$ (TD 98005; Teklad, Madison, WI, USA) throughout the course of the studies. During the study, mice were pair-fed and body weight was recorded weekly. All the mice had free access to distilled water and were fed $3 \mathrm{~g} / \mathrm{d}$ per mice of the respective diet, the minimum average food intake of the mice during the acclimatisation period. Before killing, the mice were housed individually in a metabolism cage for the collection of urine. On the day of killing, blood was collected from the orbital venous sinus of the mice. The uterus was collected, and the uterine index or uterus/body weight ratio was calculated by normalising the weight of the uterus with the final body weight of the mice. Kidneys were collected for mRNA expression detection. The bone specimens were obtained for peripheral computed tomography analysis.

Biochemical assays of serum and urine samples. Ca and inorganic $\mathrm{P}$ concentrations in serum and urine were determined by an $O$-cresolphthalein complexone method and a $p$-methylaminophenol reduction method, respectively, using commercial kits (Wako Pure Chemical Industries Limited, Osaka, Japan). Urinary Ca and P concentrations were normalised with creatinine concentration 


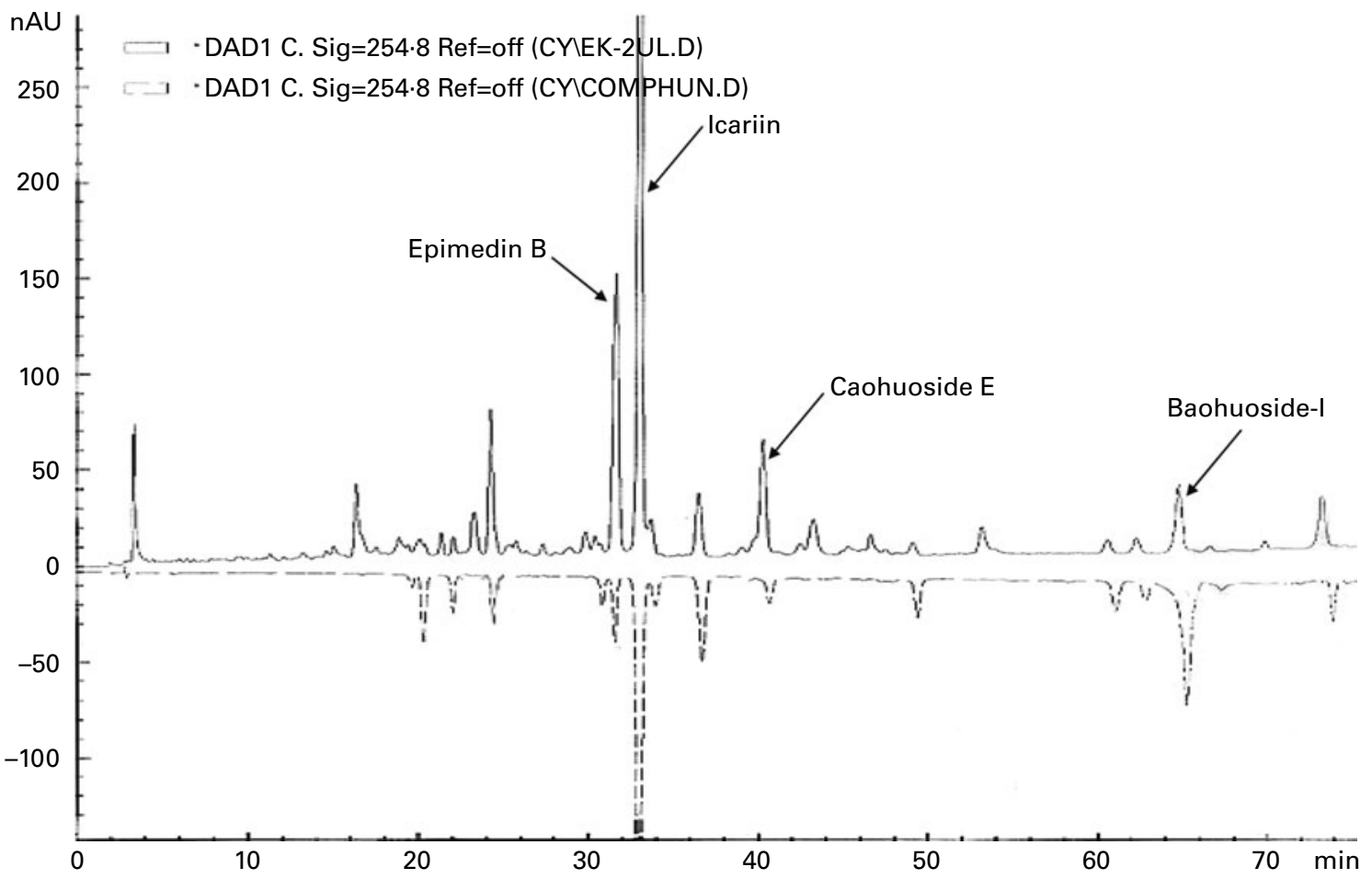

Fig. 1. Reverse-phase HPLC for the qualitative analysis of the total flavonoid extract of Herbal epimedii (HEP). Icariin, epimedin B, caohuoside E and baohuoside I are the main active compounds in HEP according to the Chinese Pharmacopoeia. HPLC analysis has been performed with standard compounds using the same elution procedure as that used with the total flavonoid (TF) extract of HEP. The peaks in the profile of TF with the same retention time with authentic markers were identified and used for confirmation of the identity of the TF extract HEP.

and were expressed as urinary $\mathrm{Ca}$ to creatinine ratio and urinary $\mathrm{P}$ to creatinine ratio, respectively.

Bone mineral density analysis by peripheral quantitative computed tomography. Peripheral quantitative computed tomography scanning was performed using XCT-2000 (StraTec Medizintechnik GmbH, Durlacher, Germany). Femurs were placed on a plastic holder and oriented at the centre of the scanning area. The long axis of diaphysis was adjusted parallel to the scanning direction. At a distance of $1.5 \mathrm{~mm}$ away from the apex of femur, proximal and distal ends were scanned at a voxel size of $0.3 \mathrm{~mm}^{2}$. BMD (in $\mathrm{mg} / \mathrm{cm}^{3}$ ) and polar stress-strain index (polar-SSI) (in $\mathrm{mm}^{3}$ ) of femur were measured. SSI is an index for the estimation of torsional bone strength ${ }^{(26)}$.

RNA isolation from kidney and quantitative real-time $R T-P C R$. Whole kidney was harvested and immediately frozen in liquid $\mathrm{N}_{2}$ and stored at $-80^{\circ} \mathrm{C}$. Frozen kidney was thawed in Trizol reagent and homogenised. Total RNA was isolated from kidney using Trizol according to the manufacturer's protocol. Total RNA was reverse transcribed using the High-Capacity complementary DNA Reverse Transcription Kit (Applied Biosystems, Inc., Foster City, CA, USA) at $25^{\circ} \mathrm{C}$ for $10 \mathrm{~min}, 37^{\circ} \mathrm{C}$ for $2 \mathrm{~h}$ and $85^{\circ} \mathrm{C}$ for $5 \mathrm{~s}$. The sequences of the PCR primers were 5'-TCCACGATGGATCTGAATGA-3' (vitamin D receptor (VDR) forward) and 5'-GCAGCACATGRRCTTCCTCA-3' (VDR reverse) for VDR, 5'-ATGCCAGCAACTGAAGTCCT-3' (CaBP-28K forward) and 5'-AGCAAAGCATCCAGCTCATT$3^{\prime}$ (CaBP-28K reverse) for CaBP-28K, and 5'-GACCACAGT-
CCATGCCATCAC-3' (glyceraldehyde-3-phosphate dehydrogenase (GAPDH) forward) and 5'-GCTGTTGAAGTCGCAGGAGAC-3' (GAPDH reverse) for the housekeeping gene GAPDH. PCR was carried out in $20 \mu \mathrm{l}$ reaction mixture containing $10 \mu \mathrm{l} \mathrm{iQ}^{\mathrm{TM}}$ SYBR Green Supermix (Bio-Rad Laboratories, Hercules, CA, USA) and $0.5 \mu \mathrm{l}$ of complementary DNA template using an ABI 7900HT Fast Real-Time PCR system (Applied Biosystems, Inc.). The following cycle parameters were used: one cycle of $95^{\circ} \mathrm{C}$ for $1 \mathrm{~min}$ and forty cycles of $95^{\circ} \mathrm{C}$ for $20 \mathrm{~s}$; different melting temperature for $20 \mathrm{~s}$ and $72^{\circ} \mathrm{C}$ for $18 \mathrm{~s}$. Upon completion, a melting curve was examined. Standard curves were generated using serially diluted solutions of complementary DNA derived from control sample. The target gene transcripts in each sample were normalised on the basis of its GAPDH.

Expt 2: bone anabolic effect of total flavonoid at optimal dosage in ovariectomised mice

Experimental groupings. Based on the results of Expt 1, the optimised dosage of $\mathrm{TF}(100 \mu \mathrm{g} / \mathrm{g}$ per $\mathrm{d})$ for increasing total BMD and polar SSI at distal femur as well as renal CaBP-28K and VDR mRNA expression was chosen for the second study. One-month-old C57BL/6J mice were randomly separated into four groups including Sham + vehicle (Sham, $n 8)$, OVX + vehicle $(n 8)$, OVX $+\mathrm{E}_{2}(n 8)$ and OVX + TF (TF100, $100 \mu \mathrm{g} / \mathrm{g}$ per $\mathrm{d}, n$ 8). After recovery from surgery for $18 \mathrm{~d}$, they were orally administered with 
vehicle, $E_{2}(4 \mu \mathrm{g} / \mathrm{g}$ per d) and TF $(100 \mu \mathrm{g} / \mathrm{g}$ per d) for 6 weeks.

Bone microarchitecture analysis by microCT. Right femurs from mice were scanned using the microCT system (viva-CT40; Scanco Medical, Bassersdorf, Switzerland) to evaluate trabecular bone volume fraction and microarchitecture in the epiphysis region of the femur end. The scanning position of distal femur was initiated at the distal end of the femur. All scans were done in $21 \mu \mathrm{m}$ sections to a total of 100 slices per scan. Raw data generated were reconstructed to the image files. The two-dimensional and three-dimensional evaluation can be generated by the Scanco compiler in the Virtual Memory System. Different threshold values were chosen for binarising the trabecular and cortical bones, and the volume of interest was contoured in twenty serial slices in 100 scanned slices for evaluation. The trabecular and the cortical parts of the distal femur were separated with semi-automatically drawing contours. Only trabecular bones were contoured, and the threshold value is set as $210 \mathrm{mg}$ hydroxyapatite $/ \mathrm{cm}^{3}$. After evaluations, five microstructural parameters were used in the data analysis such as bone volume/tissue volume, trabecular number, trabecular thickness, trabecular separation and structural model index.

RNA isolation from bone and quantitative real-time $R T-P C R$. Whole left femur was harvested and immediately frozen in liquid $\mathrm{N}_{2}$ and stored at $-80^{\circ} \mathrm{C}$. Frozen femur was put in an RNase-free mortar and pestle which contained liquid $\mathrm{N}_{2}$, and then grinded into a fine pieces immersed in liquid $\mathrm{N}_{2}$. Then, the frozen powders were transferred into a tube containing Trizol. Total RNA was extracted, and the gene expression of OPG, RANKL, type I collagen, osteocalcin, IL-6 and GAPDH was assessed as described in Expt 1. The sequences of the PCR primers were 5'-TGAGTGTGAGGAAGGGCGTTA-3' (OPG forward) and $5^{\prime}$-CCATCTGGACATTTTTTGCAAA-3' (OPG reverse), 5'-GCACACCTCACCATCAATGCT-3' ${ }^{\prime}$ (RANKL forward)

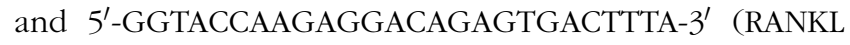
reverse), 5'-CTTGGTGGTTTTGTATTCGATGAC-3' (type I collagen forward) and $5^{\prime}$-GCGAAGGCAACAGTCGCT- $3^{\prime}$ (type I collagen reverse), 5'-CTCACAGATGCCAAGCCCA$3^{\prime}$ (osteocalcin forward) and 5'-CCAAGGTAGCGCCGGAGTCT-3' (osteocalcin reverse), 5'-TGGGAAATCGTGGAAATGAGA- $3^{\prime}$ (IL-6 forward) and 5'-CAAGTGCATCATCGTTGTTCATAC- $3^{\prime}$ (IL-6 reverse), and 5'-GACCACAGTCCATGCCATCAC-3' (GAPDH forward) and 5'-GCTGTTGAAGTCGCAGGAGAC-3' (GAPDH reverse), respectively.

\section{Statistical analysis}

The data were analysed by one-way ANOVA, and followed by Tukey's multiple comparison test as a post test to compare the group means if overall $P<0 \cdot 05$. GraphPad Prism (El Camino Real, CA, USA) version 4.4 software was used. The results were reported as means with their standard

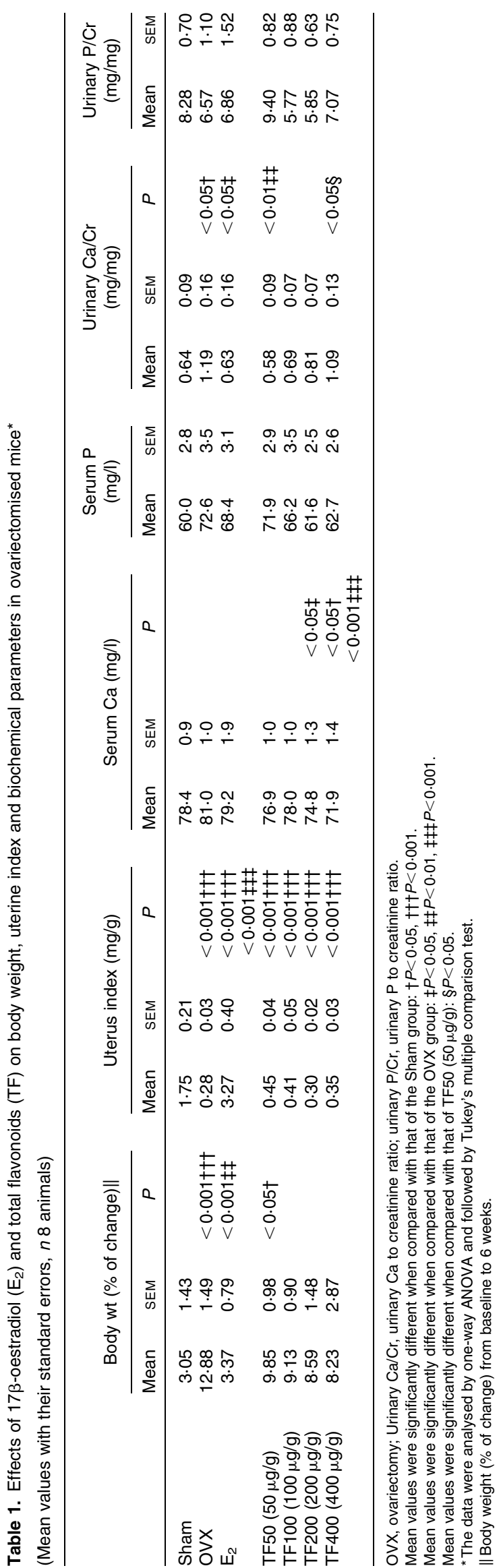


errors. A $P$-value $<0.05$ was considered statistically significant.

\section{Results}

Dose-dependent effects of total flavonoid on body weight, uterine index and biochemical parameters in ovariectomised mice

Body weight increased significantly in the OVX group and $50 \mu \mathrm{g} / \mathrm{g}$ TF group. Oestradiol, but not TF, prevented the OVX-induced increase in weight gain in mice (Table 1). The uterine index was significantly reduced in OVX mice, suggesting that the surgery was successful. In contrast to $\mathrm{E}_{2}$, TF did not increase the uterus index in OVX mice (Table 1). Serum Ca levels were not significantly altered by ovariectomy or treatment with $\mathrm{E}_{2}$, or 50 and $100 \mu \mathrm{g} / \mathrm{g}$ TF; while 200 and $400 \mu \mathrm{g} / \mathrm{g}$ TF decreased serum Ca levels in OVX mice ( $v$. OVX, $P<0.05$ ). Urinary $\mathrm{Ca}$ excretion was suppressed in OVX mice treated with $\mathrm{E}_{2}$ and $\mathrm{TF}$ at $50 \mu \mathrm{g} / \mathrm{g}(v$. OVX, $P<0.05$ and 0.01 , respectively). The suppression of urinary $\mathrm{Ca}$ excretion by $\mathrm{TF}$ in OVX mice was found to be dose dependent. There were no statistically significant differences in serum $\mathrm{P}$ level and urinary $\mathrm{P}$ excretion between each group.

Dose-dependent effects of total flavonoid on bone mineral density and bone strength in femur of ovariectomised mice

The effects of OVX, $\mathrm{E}_{2}$ and TF on bone mass and SSI at distal femur are presented in Table 2. Ovariectomy decreased total BMD $(-11 \%)$, trabecular BMD (-13\%) and polar SSI $(-53 \%)$ at distal femur in mice. Treatment of OVX mice with $\mathrm{E}_{2}$ significantly increased total BMD, trabecular BMD as well as polar SSI at distal femur by $40 \%$ $(P<0.001), 56 \%(P<0.001)$ and $228 \%(P<0.001)$, respectively, when compared with OVX mice. TF increased total BMD, and the most effective dosages were 50 and $100 \mu \mathrm{g} / \mathrm{g}(v$. OVX), but the increase did not reach statistical significance. TF also increased the trabecular BMD with the most effective dosage at $50 \mu \mathrm{g} / \mathrm{g}(v$. OVX, $P<0 \cdot 05)$. Treatment of OVX mice with TF (50 and $100 \mu \mathrm{g} / \mathrm{g}$ ) dramatically increased polar SSI at the distal femur by $119 \%(P<0 \cdot 001)$ and $131 \%(P<0 \cdot 001)$, respectively.

\section{Dose-dependent effects of total flavonoid on vitamin $D$ receptor and calcium transport protein mRNA expression in kidney}

1,25-Dihydroxyvitamin $\mathrm{D}_{3}$ regulates Ca absorption through acting on the epithelial Ca-transporting proteins primarily via a genomic action after binding with its receptor (VDR). CaBP-28K is the major vitamin D-dependent CaBP-28K expressed in the kidney ${ }^{(27)}$. To determine whether the suppression of urinary $\mathrm{Ca}$ excretion by TF was associated with the changes in renal expression of major proteins involved in renal $\mathrm{Ca}$ transport, renal expression of CaBP-28K and VDR mRNA was determined. As shown in Fig. 2, ovariectomy did not alter renal expression of CaBP-28K and VDR mRNA in mice. $\mathrm{E}_{2}$ significantly increased renal VDR mRNA, but not CaBP-28K expression, in OVX mice ( $v$. OVX, $P<0.05)$. TF increased CaBP-28K and VDR mRNA expression in OVX mice, and the maximal effect was at 100 and $50 \mu \mathrm{g} / \mathrm{g}$, respectively.

\section{Effects of total flavonoid on bone microarchitecture at distal femur in ovariectomised mice analysed by microCT}

The present results showed that the maximal bone-protective effects on total BMD, trabecular BMD as well as polar SSI were between 50 and $100 \mu \mathrm{g} / \mathrm{g}$ of TF of HEP, and the optimal stimulating effect on renal CaBP-28K and VDR mRNA expression was also between 50 and $100 \mu \mathrm{g} / \mathrm{g}$ of TF. Based on these results, $100 \mu \mathrm{g} / \mathrm{g}$ of $\mathrm{TF}$ was chosen for subsequent experiments. The bone-protective effects of $\mathrm{E}_{2}$ and $\mathrm{TF}$ on bone microarchitecture at distal femur of OVX mice were clearly illustrated by their three-dimensional images obtained from microCT analysis as shown in Fig. 3(a). Ovariectomy significantly decreased bone

Table 2. Effects of 6-week treatment of $17 \beta$-oestradiol $\left(E_{2}\right)$ and total flavonoids (TF) on bone mineral density and bone strength at distal femur in ovariectomised mice analysed by peripheral quantitative computed tomography*

(Mean values with their standard errors, $n$ 6-8 animals)

\begin{tabular}{|c|c|c|c|c|c|c|c|c|c|}
\hline & \multicolumn{3}{|c|}{ Total BMD (mg/ccm) } & \multicolumn{3}{|c|}{ Trabecular BMD (mg/ccm) } & \multicolumn{3}{|c|}{ Polar SSI $\left(\mathrm{mm}^{3}\right)$} \\
\hline & Mean & SEM & $P$ & Mean & SEM & $P$ & Mean & SEM & $P$ \\
\hline OVX & $347 \cdot 3$ & $9 \cdot 6$ & & $342 \cdot 6$ & $8 \cdot 3$ & $<0.05 \dagger$ & 0.32 & 0.05 & $<0.01 \dagger \dagger$ \\
\hline $\mathrm{E}_{2}$ & $489 \cdot 4$ & $9 \cdot 6$ & $\begin{array}{l}<0.001 \dagger \dagger \dagger \\
<0.001 \ddagger \ddagger \ddagger\end{array}$ & $536 \cdot 1$ & $10 \cdot 9$ & $\begin{array}{l}<0.001 \dagger † \dagger \\
<0.001 \ddagger \ddagger \ddagger\end{array}$ & $1 \cdot 05$ & 0.06 & $\begin{array}{l}<0.001 \dagger \dagger \dagger \\
<0.001 \ddagger \ddagger \ddagger\end{array}$ \\
\hline TF50 $(50 \mu \mathrm{g} / \mathrm{g})$ & $387 \cdot 7$ & 9.5 & & $396 \cdot 4$ & 11.5 & $<0.05 \ddagger$ & 0.70 & 0.07 & $<0.001 \neq \ddagger \ddagger$ \\
\hline TF100 $(100 \mu \mathrm{g} / \mathrm{g})$ & $387 \cdot 1$ & $10 \cdot 0$ & & $384 \cdot 1$ & $11 \cdot 3$ & & 0.74 & 0.06 & $<0.001 \ddagger \ddagger \ddagger$ \\
\hline TF200 $(200 \mu \mathrm{g} / \mathrm{g})$ & $371 \cdot 0$ & $6 \cdot 0$ & & $376 \cdot 3$ & $6 \cdot 2$ & & 0.60 & 0.03 & \\
\hline TF400 $(400 \mu \mathrm{g} / \mathrm{g})$ & $367 \cdot 1$ & $7 \cdot 6$ & & $364 \cdot 2$ & $7 \cdot 4$ & & 0.54 & 0.06 & \\
\hline
\end{tabular}

$\mathrm{BMD}$, bone mineral density; SSI, stress-strain index; OVX, ovariectomy.

Mean values were significantly different when compared with that of the Sham group: $\dagger P<0.05, \dagger \dagger P<0.01, \dagger \dagger \dagger P<0.001$.

Mean values were significantly different when compared with that of the OVX group: $\ddagger P<0.05, \ddagger \ddagger \ddagger P<0.001$.

* The data were analysed by one-way ANOVA and followed by Tukey's multiple comparison test. 
(a)

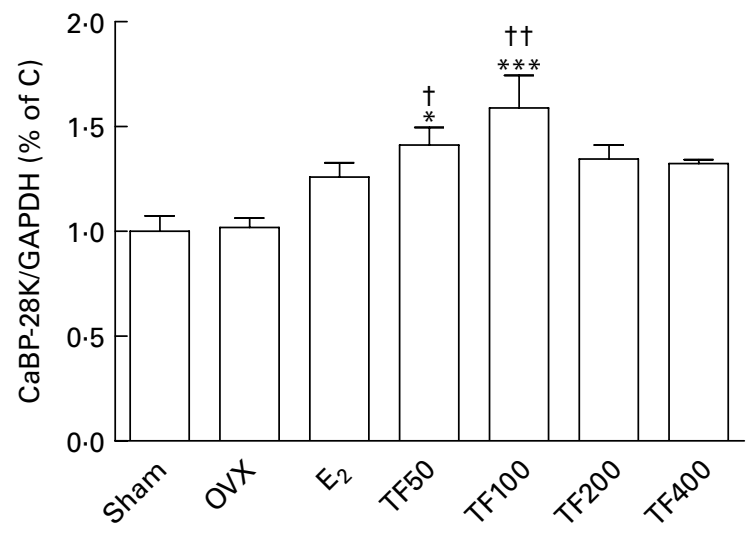

(b)

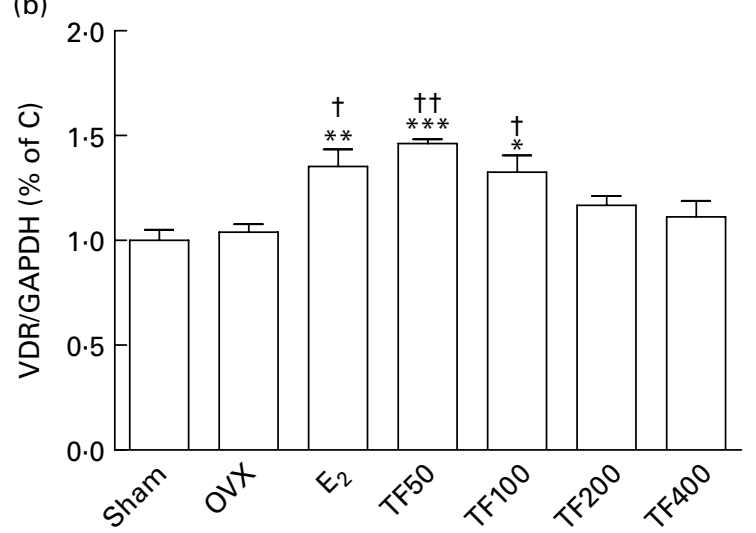

Fig. 2. Effects of $17 \beta$-oestradiol $\left(E_{2}\right)$ and total flavonoid (TF) on Ca transport protein (CaBP-28K) and vitamin $D$ receptor (VDR) mRNA expressions in kidney. Ovariectomy (OVX) mice were treated with vehicle (Sham or OVX), $E_{2}$ $(4 \mu \mathrm{g} / \mathrm{g}$ per d) or four doses of TF (TF50, $50 \mu \mathrm{g} / \mathrm{g}$ per d; TF100, $100 \mu \mathrm{g} / \mathrm{g}$ per d; TF200, $200 \mu \mathrm{g} / \mathrm{g}$ per $\mathrm{d}$ and TF400, $400 \mu \mathrm{g} / \mathrm{g}$ per d) for 6 weeks. At killing, kidney was collected and total RNA was isolated. Real-time RT-PCR was performed to determine the mRNA expressions of (a) CaBP-28K and (b) VDR, which were normalised with that of glyceraldehyde-3-phosphate dehydrogenase (GAPDH). The data were obtained at 6 weeks and were expressed as a percentage of the control mice treated with vehicle. Results were expressed as means with their standard errors. Mean values were significantly different when compared with that of the sham group: ${ }^{*} P<0.05,{ }^{\star \star} P<0.01,{ }^{* \star *} P<0.001$. Mean values were significantly different when compared with that of the OVX group ( $n 5-6)$ : † $P<0.05$, †† $P<0.01$.

volume/tissue volume, trabecular number, and trabecular thickness, and increased trabecular separation and structural model index when compared to the sham group. Treatment with $\mathrm{E}_{2}$ and $\mathrm{TF}$ significantly prevented the reduction in bone volume/tissue volume, trabecular number, and trabecular thickness as well as the increase in trabecular separation and structural model index in OVX mice (Fig. 3(b)-(f)).

Effects of total flavonoid on type I collagen, osteocalcin, osteoprotegerin, receptor activator of NF- $\kappa B$ ligand and IL-6 gene expressions in femur

OVX appeared to decrease the expression of type I collagen (Fig. 4(a)) and osteocalcin (Fig. 4(b)) mRNA in the femur of mice, but the decrease was NS. $\mathrm{E}_{2}$ and TF significantly increased type I collagen and osteocalcin mRNA expressions in the femur of OVX mice (Fig. 4(a) and (b)). Treatment of OVX mice with $\mathrm{E}_{2}$ significantly decreased RANKL mRNA expression (Fig. 4(d)), but did not significantly affect OPG mRNA expression (Fig. 4(c)) in femur. Both $\mathrm{E}_{2}$ and $\mathrm{TF}$ significantly increased the ratio of OPG/RANKL in the femur of OVX mice (Fig. 4(e)), suggesting that they might modulate the process of osteoclastogenesis via their actions on OPG and RANKL expression in bone cells. OVX significantly increased the gene expression of IL-6 $(P<0 \cdot 01)$. TF, but not $\mathrm{E}_{2}$, treatment significantly decreased the IL-6 gene expression in the femur of OVX mice $(P<0.05)$.

\section{Discussion}

The present study clearly demonstrated that the TF of HEP could suppress OVX-induced increase in urinary $\mathrm{Ca}$ excretion as well as loss in bone mass and bone strength in mice in a dose-dependent manner. In addition, renal expressions of CaBP-28K and VDR mRNA were dose dependently induced by treatment with TF in OVX mice. Furthermore, TF could improve trabecular microarchitecture in OVX mice, and significantly increased the mRNA expression of type I collagen, osteocalcin and OPG/ RANKL ratio and suppressed IL-6 mRNA expression in the femur of OVX mice.

The present study demonstrated that the effects of TF on bone mass in OVX mice were dose dependent. It increased total BMD and trabecular BMD of the distal femur in OVX mice with the most effective dosages between 50 and $100 \mu \mathrm{g} / \mathrm{g}$. Moreover, the present study demonstrated that the effects of TF on torsional bone strength (polar SSI) at distal femur in OVX mice were also dose dependent, and the most effective doses were also between 50 and $100 \mu \mathrm{g} / \mathrm{g}$. It was of interest to note that higher concentration of $\mathrm{TF}(200$ and $400 \mu \mathrm{g} / \mathrm{g}$ ) did not result in further increase in bone mass and torsional bone strength at the distal femur in OVX mice.

Oestrogen plays an important role in $\mathrm{Ca}^{2+}$ homoeostasis, and oestrogen deficiency results in a negative $\mathrm{Ca}^{2+}$ balance and bone loss in postmenopausal women ${ }^{(28,29)}$. Ovariectomy induced a significant increase in urinary Ca level, and this effect could be reversed by TF. The present results indicated that the suppression of urinary $\mathrm{Ca}$ excretion by TF was negatively associated with the increase in renal expressions of CaBP-28K and VDR mRNA in OVX mice. The most effective dosages for their expressions in kidney were 100 and $50 \mu \mathrm{g} / \mathrm{g}$, respectively. As CaBP-28K is a vitamin D-dependent CaBP-28K in the kidney, it is possible that the decrease in urinary $\mathrm{Ca}$ loss by $\mathrm{TF}$ in OVX mice might be in part mediated through an increase in renal $\mathrm{Ca}$ transport via the induction of CaBP-28K expression. In addition, the induction of VDR expression in kidney by $\mathrm{TF}$ in OVX mice might increase renal responsiveness to vitamin $\mathrm{D}$, thereby increasing vitamin 
(a)

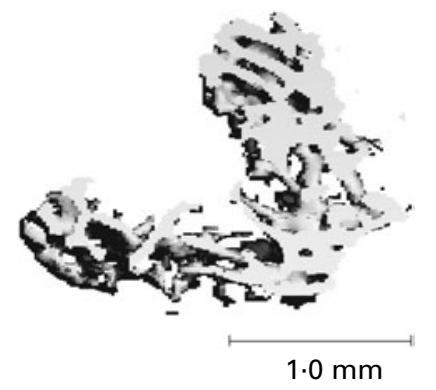

Sham

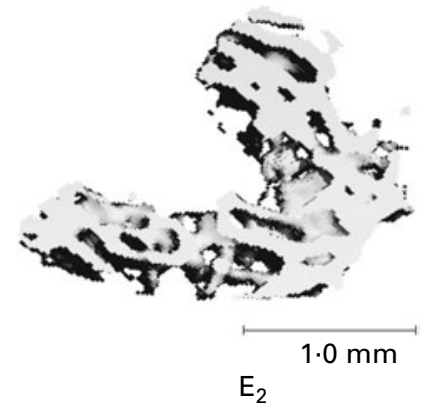

$\mathrm{E}_{2}$
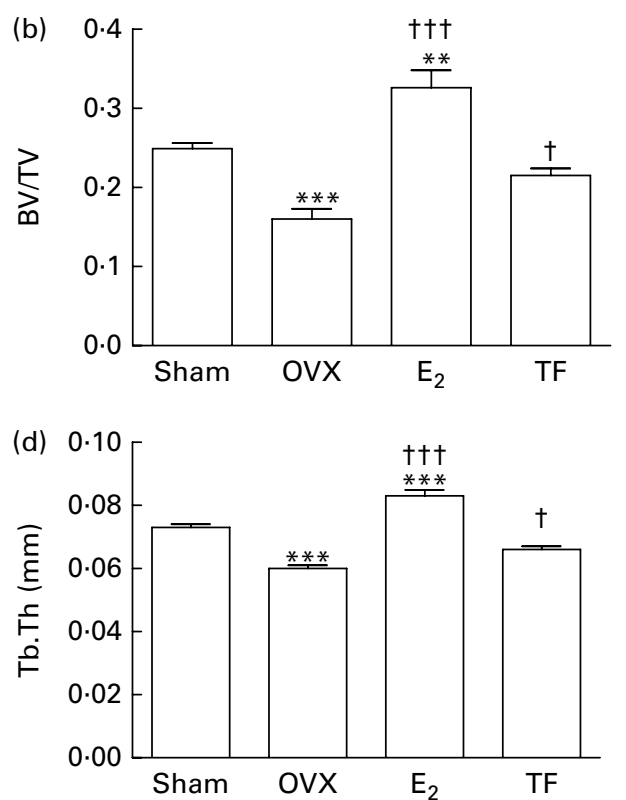
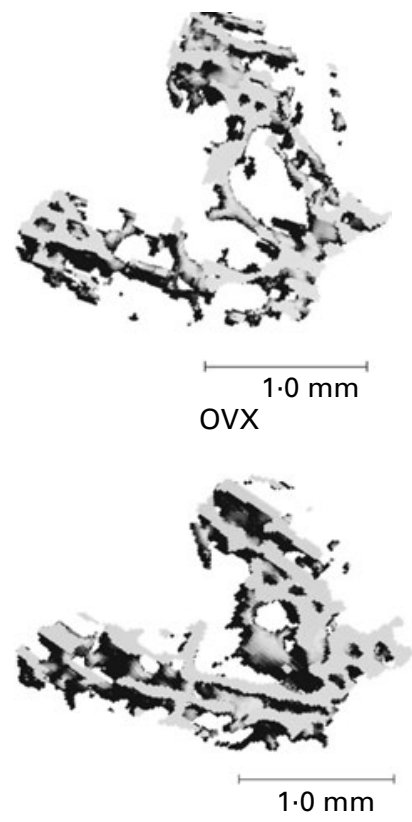

TF
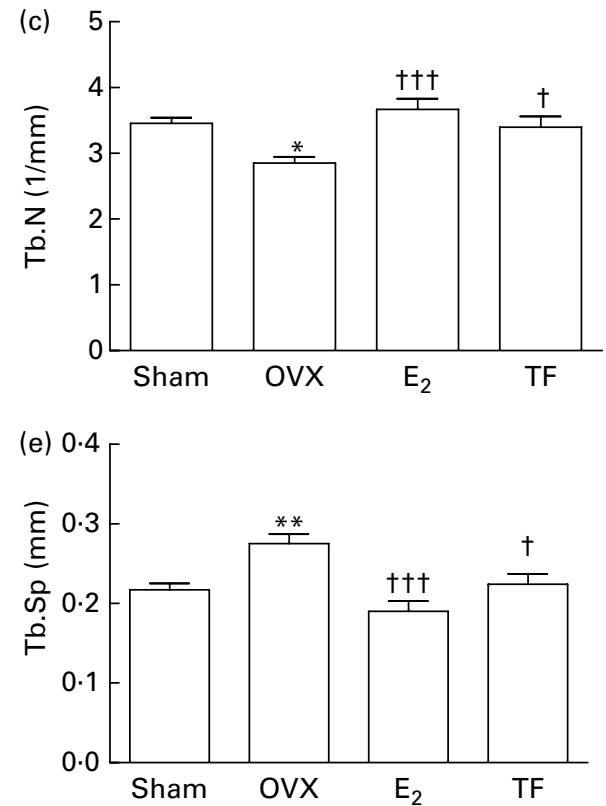

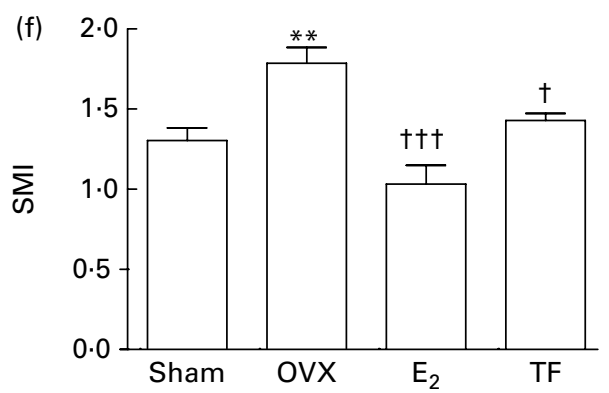

Fig. 3. Effects of $17 \beta$-oestradiol $\left(\mathrm{E}_{2}\right)$ and total flavonoid (TF) on bone microarchitecture at distal femur in ovariectomised mice analysed by microCT. Ovariectomy (OVX) mice were treated with vehicle (Sham or OVX), $E_{2}(4 \mu \mathrm{g} / \mathrm{g}$ per d) or TF $(100 \mu \mathrm{g} / \mathrm{g}$ per d) for 6 weeks. (a) Representative 3D microCT images of distal femur. Graphical measurement of bone volume/tissue volume (BV/TV) (b), trabecular number (Tb.N) (c), trabecular thickness (Tb.Th) (d), separation (Tb.Sp) (e) and structural model index (SMI) (f) as determined from the microCT. Results were expressed as means with their standard errors. Mean values were significantly different when compared with that of the Sham group: ${ }^{\star} P<0.05,{ }^{\star \star} P<0.01,{ }^{\star \star \star} P<0.001$. Mean values were significantly different when compared with that of the $\mathrm{OVX}$ group $(n 8): \dagger P<0.05, \dagger \dagger \dagger P<0.001$. 
(a)
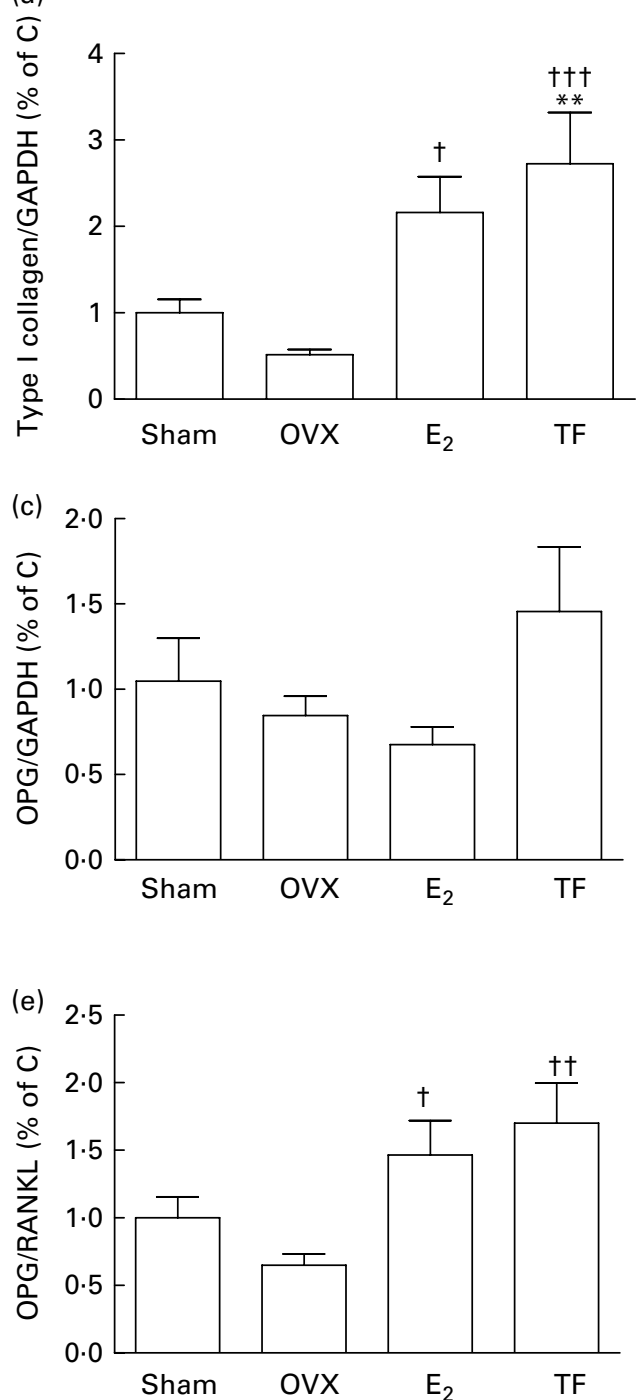

(b)
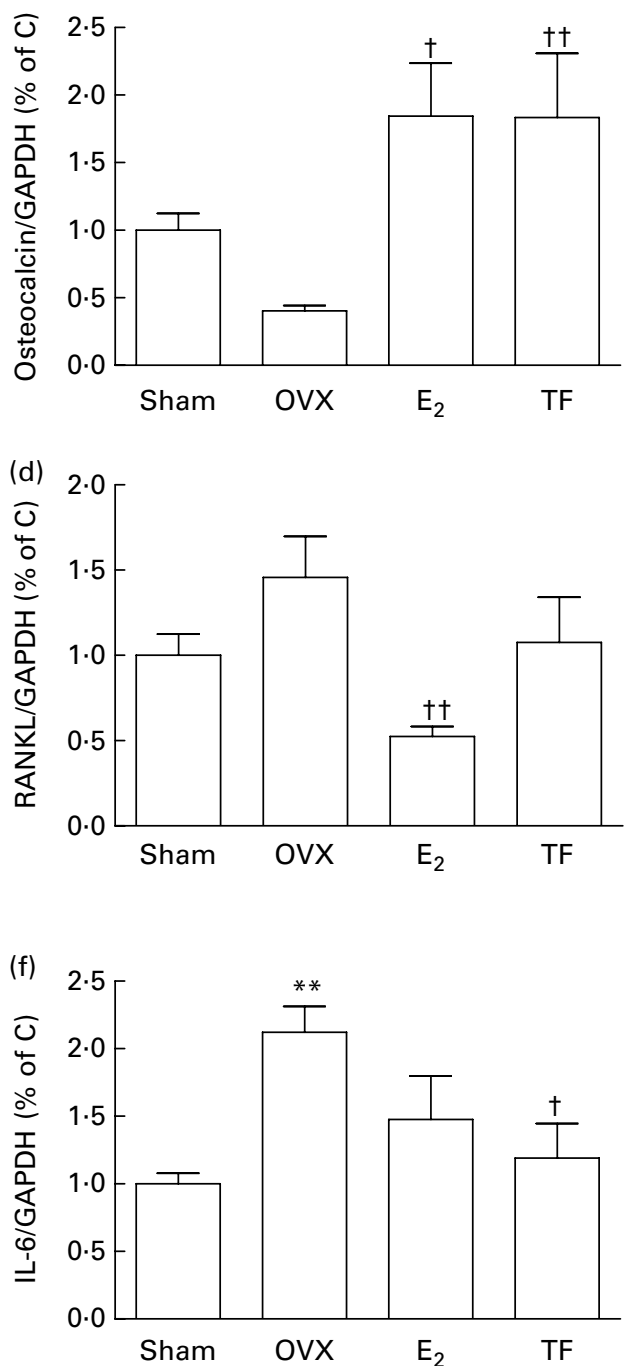

Fig. 4. Effects of $17 \beta$-oestradiol $\left(E_{2}\right)$ and total flavonoid (TF) on type I collagen, osteocalcin, osteoprotegerin (OPG), receptor activator of $N F-\kappa B$ ligand (RANKL) and IL-6 mRNA expressions in femur. Ovariectomy (OVX) mice were treated with vehicle (Sham or OVX), $E_{2}(4 \mu \mathrm{g} / \mathrm{g}$ per d) or TF (100 $\mu \mathrm{g} / \mathrm{g}$ per d) for 6 weeks. At killing, femurs were collected and total RNA was isolated. Real-time RT-PCR was performed to determine the mRNA expressions of type I collagen (a), osteocalcin (b), OPG (c), RANKL (d), OPG/RANKL (e) and IL-6 (f), which were normalised with that of glyceraldehyde-3-phosphate dehydrogenase (GAPDH). The data were obtained at 6 weeks and were expressed as a percentage of the control mice treated with vehicle. Results were expressed as means with their standard errors. Mean values were significantly different when compared with that of the Sham group: ${ }^{\star \star} P<0 \cdot 01$. Mean values were significantly different when compared with that of the OVX group $(n 5-8): \uparrow P<0.05, \dagger \dagger P<0.01, \dagger \dagger \uparrow P<0.001$

D-dependent expression of CaBP-28K in kidneys. Further study will be needed to confirm whether TF will increase renal $\mathrm{Ca}$ transport in OVX mice. Nonetheless, the present study indicated that TF not only exerts protective effects in bone tissues but also exerts additional effects on the mRNA expression of CaBP-28K in kidney, which lead to the conservation of bone mass in OVX mice.

Ovariectomy produced apparent deterioration of trabecular three-dimensional microarchitecture in mice. The present study showed that TF treatment prevented OVXinduced deterioration of microstructural parameters at the distal femur in mice. Specifically, TF at $100 \mu \mathrm{g} / \mathrm{g}$ per $\mathrm{d}$ could restore the loss of bone volume/tissue volume, trabecular number and trabecular thickness while suppressing the increase in trabecular separation and structural modulus index at distal femur in OVX mice. These results indicated that $\mathrm{TF}$ was effective not only in preserving bone mass but also in preventing the deterioration of bone microarchitecture associated with oestrogen deficiency in mice.

The study of the changes in femur gene expression in response to TF provides insights to understand its mechanism of actions involved in improvement of bone quality in OVX mice. Type I collagen and osteocalcin are the most abundant extracellular proteins produced by osteoblasts in bone and are essential for maintaining bone strength $^{(30,31)}$. TF could significantly increase type I collagen and osteocalcin mRNA expression in femur of OVX 
mice to a level comparable with those treated with oestradiol. These in vivo results are in agreement with previous reports by us ${ }^{(20)}$ and others ${ }^{(15-19)}$ that HEP could enhance bone formation through its action on cells in the osteoblastic lineage. The equilibrium of OPG and RANKL expression plays an important role in controlling bone remodelling ${ }^{(32)}$. The secretion of OPG by osteoblastic cells could block the interaction of RANKL with its functional receptor RANK expressed on the osteoclastic cell surface, thereby inhibiting osteoclastogenesis. The present results revealed that both oestradiol and TF increase the ratio of OPG/RANKL mRNA expression in the femur of OVX mice, suggesting that both agents can inhibit the process of osteoclastogenesis in vivo. However, the mechanism by which TF modulates osteoclastogenesis might be different from that of oestradiol. The results indicated that the increase in the OPG/RANKL ratio by TF was due to its inductive effects on OPG mRNA expression, while the increase by oestradiol was due to its suppressive effect on RANKL mRNA expression in vivo.

IL-6 is known to be a potent stimulator of bone resorption, and plays an important role in the induction of osteoclastogenesis and bone loss upon oestrogen depletion $^{(33,34)}$. The present results indicated that IL-6 mRNA in the femur of mice appeared to be significantly increased with ovariectomy and decreased with TF treatment in OVX mice. These results were in agreement with those reported previously by Wang et $a l^{(8)}$ in which HEP increased IL-6 mRNA in bone of OVX rats. Thus, TF might suppress the process of osteoclastogenesis not only via the induction of the OPG/RANKL ratio but also via the inhibition of the OVX-induced increase in levels of IL-6 in bone.

In search for safe and effective alternatives for treatment of postmenopausal osteoporosis, HEP has received much attention in recent years to demonstrate its efficacy as well as mechanism of actions ${ }^{(7-19,22-24)}$. In the present study, we have demonstrated that the optimal dosage of TF for improving bone mass and bone strength as well as for decreasing urinary $\mathrm{Ca}$ excretion in OVX mice was between 50 and $100 \mu \mathrm{g} / \mathrm{g}$ per $\mathrm{d}$. The present results showed that a further increase in the dosages of TF would compromise its positive effects on bone mass as well as its suppressive effects on urinary $\mathrm{Ca}$ excretion. The optimal dosages reported in the present study were in agreement with the dosages used in other reported studies using OVX rats as a model ${ }^{(12-14)}$. For example, the study by Songlin et $a l .^{(14)}$ showed that treatment of 11-month-old OVX rats with a flavonoid fraction of HEP at $10 \mathrm{mg} / \mathrm{kg}$ per $\mathrm{d}$ for 12 weeks increased BMD as well as improved bone microarchitecture. The dosage used in their studies is close to the optimal dosages reported in the present study, in which the equivalent dose in rats will be $25-50 \mathrm{mg} / \mathrm{kg}$ per $\mathrm{d}$ or $\mu \mathrm{g} / \mathrm{g}$ per $\mathrm{d}$. Furthermore, the present study was the first to report that TF also exerts additional effects on the mRNA expression of
CaBP-28K in kidney, which potentially led to the suppression of urinary $\mathrm{Ca}$ excretion and the conservation of bone mass in OVX mice.

The present study clearly demonstrated that the TF of HEP could protect against bone loss and bone deterioration associated with oestrogen deficiency without exerting uterotrophic effects. Gene expression studies indicated that TF treatment achieves its osteoprotective actions in vivo via the modulation of renal Ca transport, osteoblastic functions, the process of osteoclastogenesis as well as osteoclastic functions in OVX mice. The present study showed that TF is the active fraction in HEP and defines the optimal dosages of HEP for achieving boneprotective actions in vivo.

\section{Acknowledgements}

The present work was supported by the Areas of Excellence Scheme established under the University Grants Committee of the Hong Kong Special Administrative Region (HKSAR), People's Republic of China (AOE/P-10/ 01), the Hong Kong Polytechnic University Central Research Fund (GYE-47), the Earmarked Research Grant Council (PolyU 5402/04M, N_PolyU536/04, PolyU 563706), HKSAR and the National Natural Science Foundation of China (30971004). We thank the support of the State Key Laboratory of Chinese Medicine and Molecular Pharmacology, HKSAR. W.-F. C. and S.-K. M. performed the majority of the laboratory work and contributed to the analysis of data and the writing of the manuscript, and contributed equally to the studies. X.-L. W. performed the preparation of the TF fraction from HEP total extract. K.-H. L. and W.-P. L. conducted some of the laboratory work. H.-K. L. performed bone microarchitecture analysis by using microCT. P.-C. L. played a significant role in the design of the study. X.-S. Y. was a co-investigator and grant holder, and played a significant role in the design and performance of the study. M.-S. W. was the principal investigator and grant holder, and played a major role in the design and performance of the study, interpretation of the results and the writing of the paper. The authors have no conflicts of interest to declare.

\section{References}

1. Gambacciani M, Ciaponi M \& Genazzani AR (2007) The HRT misuse and osteoporosis epidemic: a possible future scenario. Climacteric 10, 273-275.

2. Rossouw JE, Anderson GL, Prentice RL, et al. (2002) Writing Group for the Women's Health Initiative Investigators. Risks and benefits of estrogen plus progestin in healthy postmenopausal women: principal results from the women's health initiative randomized controlled trial. JAMA 288, 321-333.

3. Million Women Study Collaborators (2003) Breast cancer and hormone-replacement therapy in the Million Women Study. Lancet 362, 419-427.

4. Papaioannou A, Kennedy CC, Dolovich L, et al. (2007) Patient adherence to osteoporosis medications: problems, 
consequences and management strategies. Drugs Aging 24 $37-55$.

5. An SJ, Li T \& Li E (2002) Effect of kidney-tonifying herbs on ovary function and bone mass in postmenopausal women. Chin J Osteoporosis 6, 55-59.

6. Wang JS, Xu XJ, Jin JS, et al. (1997) Clinical study of treatment of female osteoporosis with Yishen Jiangu pills. Chin J Osteoporosis 3, 1-63.

7. Yu S, Chen K, Li S, et al. (1999) In vitro and in vivo studies of the effect of a Chinese herb medicine on osteoclastic bone resorption. Chin J Dent Res 2, 7-11.

8. Wang BL, Quan JX, Guo SY, et al. (2000) Effects of Epimedium on the expression of interleukin- 6 mRNA in bone of OVX rat. Chin J Obstet Gynecol 35, 724-726.

9. Ji H, Liu K, Gong XJ, et al. (2001) Effect of Epimedium koreanum favoids on osteoporosis in ovariectomized rats. Chin J Osteoporosis 7, 4-8.

10. Jiang YN, Mo HY \& Chen JM (2002) Effects of Epimedium total flavonoids phytosomes on preventing and treating bone loss of ovariectomized rats. Zhongguo Zhong Yao Za Zbi 27, 221-224.

11. Sun Y, Lee SM, Wong YM, et al. (2008) Dosing effects of an antiosteoporosis herbal formula - a preclinical investigation using a rat model. Phytother Res 22, 267-273.

12. Qian C, Zhang X, Lu L, et al. (2006) Regulation of Cbfa 1 Expression by total flavonoids of Herba epimedii. Endocr J 53, 87-94.

13. Chen BL, Xie DH, Wang ZW, et al. (2009) Effect of total flavone of Epimedium on expression of bone OPG, OPGL mrRNA in ovariectomized rats. Zhongguo Gu Shang 22, $271-273$.

14. Songlin P, Ge Z, Yixin H, et al. (2009) Epimedium-derived flavonoids promote osteoblastogenesis and suppress adipogenesis in bone marrow stromal cells while exerting an anabolic effect on osteoporotic bone. Bone 45, 534-544

15. Han L-M, Liu B \& Xu P (2003) Influence of Herba epimedii flavone on proliferation of osteoblast. Shanghai J Trad Chin Med 37, 56-58.

16. Wang JQ, Hu YG, Zheng HJ, et al. (2002) The effect of icariin on proliferation and differentiation of osteoblasts in vitro. Chin J Clin Rehab 6, 1037-1038.

17. Li Y, Ji H, Li P, et al. (2002) Effects of Epimedium pubescens flavonoids on osteoblast in vitro. J China Pharm Univ 33, $48-50$.

18. Zhang DW, Cheng Y, Wang NL, et al. (2008) Effects of total flavonoids and flavonol glycosides from Epimedium koreanum Nakai on the proliferation and differentiation of primary osteoblasts. Phytomedicine 15, 55-61.

19. Zhang JF, Li G, Chan CY, et al. (2010) Flavonoids of Herba Epimedii regulate osteogenesis of human mesenchymal stem cells through BMP and Wnt/ $\beta$-catenin signaling pathway. Mol Cell Endocrinol 341, 70-74.
20. Xie F, Wu CF, Lai WP, et al. (2005) The osteoprotective effect of Herba Epimedii (HEP) extract in vivo and in vitro. Evid Based Complement Alternat Med 2, 353-361.

21. Zhang G, Qin L \& Shi Y (2007) Epimedium-derived phytoestrogen flavonoids exert beneficial effect on preventing bone loss in late postmenopausal women: a 24-month randomized, double-blind and placebo-controlled trial. J Bone Miner Res 22, 1072-1079.

22. Huang J, Yuan L, Wang X, et al. (2007) Icaritin and its glycosides enhance osteoblastic, but suppress osteoclastic, differentiation and activity in vitro. Life Sci 81, 832-840.

23. Chen KM, Ge BF, Liu XY, et al. (2007) Icariin inhibits the osteoclast formation induced by RANKL and macrophagecolony stimulating factor in mouse bone marrow culture. Pharmazie 62, 388-391.

24. Mok SK, Chen WF, Lai WP, et al. (2010) Icariin protects estrogen deficiency induced bone loss and activates estrogen receptor dependent osteoblastic functions in UMR 106 cells. Br J Pharmacol (Epublication ahead of print version 27 January 2010).

25. Nian H, Ma MH, Nian SS, et al. (2009) Antiosteoporotic activity of icariin in ovariectomized rats. Phytomedicine $\mathbf{1 6}$, 320-326.

26. Lind PM, Lind L, Larsson S, et al. (2001) Torsional testing and peripheral quantitative computed tomography in rat humerus. Bone 29, 265-270.

27. Lambers TT, Mahieu F, Oancea E, et al. (2006) CalbindinD28K dynamically controls TRPV5-mediated $\mathrm{Ca}^{2+}$ transport EMBO J 25, 2978-2988.

28. Hoenderop JG, Nilius B \& Bindels RJ (1994) Estrogen effects on calcitropic hormones and calcium homestasis. Endocr Rev 15, 301-309.

29. Young MM \& Nordin BEC (1967) Effects of natural and artificial menopause on plasma and urinary calcium and phosphorus. Lancet 2, 118-120.

30. Mahonen A, Jukkola A, Risteli L, et al. (1998) Type I procollagen synthesis is regulated by steroids and related hormones in human osteosarcoma cells. J Cell Biochem $\mathbf{6 8}$, 151-163.

31. Power RA, Iwaniec UT \& Wronski TJ (2002) Changes in gene expression associated with the bone anabolic effects of basic fibroblast growth factor in aged ovariectomized rats. Bone 31, 143-148.

32. Bord S, Ireland DC, Beavan SR, et al. (2003) The effects of estrogen on osteoprotegerin, RANKL, and estrogen receptor expression in human osteoblasts. Bone 32, 136-141.

33. Cuzzocrea S, Mazzon E, Dugo L, et al. (2003) Inducible nitric oxide synthase mediates bone loss in ovariectomized mice. Endocrinology 144, 1098-1107.

34. Jilka R, Hangoc G, Girasole G, et al. (1992) Increased osteoclast development after estrogen loss: mediation by interleukin-6. Science 257, 88-91. 